\title{
COMPARATIVE ANALYSIS OF THE STAGED SURGICAL TREATMENT OF CHILDREN WITH CONGENITAL DEFORMITIES OF THE THORACIC SPINE AGAINST THE BACKGROUND OF MULTIPLE MALFORMATIONS OF THE VERTEBRAE
}

\author{
Vissarionov S.V., Husainov N.O., Baindurashvili A.G., \\ Kokushin D.N., Kartavenko K.A. \\ H.Turner National Medical Research Center for Children's Orthopedics \\ and Trauma Surgery of the Ministry of Health of the Russian Federation, \\ Saint-Petersburg, Russia, e-mail: turner01@mail.ru
}

Aims

The study was aimed at the comparative analysis of the efficacy of the correction of spinal deformity and changes in the hemithorax volume with the use of rib endocorrectors and spinal hardware in children with congenital deformities of the thoracic spine on the background of multiple defects of its development and synostosis of the ribs.

Materials and methods

The examination and treatment of 13 patients with spinal deformities on the background of multiple malformations was carried out. Patients were subdivided into 2 groups: in patients of group $1(n=6)$, the abnormal vertebrae were removed, the local deformation was corrected, and the spinal motion segment was stabilised; in patients of group $2(n=7)$, rib endocorrectors were placed and step-wise distraction was carried out every 6-8 months. Based on the X-ray study (radiography and MSCT), the effect of interventions on the scope of correction of scoliotic and kyphotic deformation components as well as the volume of the lungs was assessed in both groups of patients after primary intervention.

\section{Results}

After surgical treatment, the scoliotic deformity correction value in the first group of patients was $63.7 \%$, kyphotic $-18.7 \%$; in group 2 , the scoliotic deformity correction value was $8.5 \%$, kyphotic $-7.8 \%$. The total lung volume in patients of group 1 and 2 prior to treatment was $972.8 \pm 339.1$ and $990.6 \pm 399 \mathrm{~cm}^{3}$, respectively. The total lung volume in patients of group 1 and 2 after treatment was $994.8 \pm \overline{3} 46.1$ and $1,484.2 \pm 407.1 \mathrm{~cm}^{3}$, respectively.

Conclusions

It was found that congenital deformity correction of the thoracic spine in children with multiple vertebral anomalies via spinal systems is more effective than via rib endocorrectors. Radical local deformation correction and stabilisation of spinal motion segments by means of spinal hardware do not adversely affect the development of the chest organs.

Keywords: congenital scoliosis, children, correction, rib implants, lung volume.

\section{INTRODUCTION}

Surgical treatment of congenital spinal deformity in children is a complex yet urgent problem of today's orthopedics. Recent papers cover the treatment of thoracic and lumbar spine curvature in children, in whom vertebrae deformities and fusions are isolated. The papers detail the tactics and methodology of surgical treatment of such pathology $[1 ; 2]$. It should, however, be noted that treating children with congenital spinal deformity associated with multiple vertebral abnormalities is an issue yet to be resolved.

Thoracic insufficiency is one of the negative consequences that vertebral column curvature might have when coupled with multiple vertebral malformations; it might also be caused by unilateral rib synostosis, especially in the thoracic segment. This condition disables normal pulmonary venti- lation and development as the child grows; clinically, it manifests itself in the form of respiratory failure and impaired motility of the thoracic walls [3]. Mehta et al. have proven the vertebral column growth to be in direct correlation with the thorax development. Their data suggest that any pathology in any of those compartments will inevitably result in scoliotic spinal deformity, as well as in a severe thoracic deformity [4]. Karol et al., Emans et al. have confirmed the negative impact of early arthrodesis. They state that fusing over $60 \%$ of the thoracic spine in a child aged 8 or younger may reduce the pulmonary function to $50 \%$ of its normal value $[5 ; 6]$. Thus, a surgery of spinal deformity coupled with multiple malformations should always seek to fully fix the deformity while not restraining spinal growth [7]. 
Metal structures have been designed to that end that enable hemithoracic growth on the concave side of the deformity (costal block osteotomy is performed in many cases for that reason). Implanting such a structure does not require any intervention on the spine itself. This enables spinal growth and further development of thoracic organs [8]. The VEPTR (vertical expandable prosthetic titanium rib) was the first such structure to be proposed and gain traction [3]. Clinical studies demonstrated mediated reduction in spinal deformity, improved external respiration as shown by treadmill tests and blood $\mathrm{Hg}$ level tests, improved SAL (space available for the lung) or other X-ray readings $[3 ; 9 ; 10]$. However, Redding et al. believe that a larger hemithorax does not equal better external breathing [11]. At the same time, Mehta et al. suggested that a metal implant placed to limit the rib excursion, coupled with the postoperative scarring, would impact external respiration as adversely as the deformity itself [4]. There are reports of a spontaneous vertebral fusion occurring in patients subjected to staged surgeries using such structures; reports indicate that such surgeries have a negative impact on the frontal torso balance or may cause kyphosis of the sagittal profile [12]. A significant drawback of such surgeries is that they are prone to complications that occur in $32 \%$ to $72 \%$ of all cases, mainly due to the loosening of metal supports, poor healing of the operative wounds due to an infection, and pleural wounding [13].

Thus, the existing data suggest the problem remains complicated, with no universal treatment, while surgical intervention remains questionably effective when it comes to treating congenital spinal deformity coupled with multiple vertebral development abnormalities and costal synostosis in children. Besides, there are no studies that have compared two fundamentally different methods: spinal systems and costal implants.

The goal hereof was to compare the performance of spinal deformity treatment and hemithorax volume adjustment by means of costal implants and spinal metal structures in children with congenital thoracic spine deformity coupled with multiple spinal malformations and costal synostosis.

\section{MATERIALS AND METHODS}

The study involved 13 patients (8 girls and 5 boys) aged 33 months to 5 years; all of them had congenital thoracic spine deformity coupled with multiple vertebral malformations; the children were treated surgically at the Spinal Pathology and Neurosurgery Unit, Turner Scientific Research Institute for Children's Orthopedics.

The study protocol followed guidelines for experimental investigation with human subjects in accordance with the Declaration of Helsinki and was approved by the ethics committee. Written informed consent was obtained from each patient (or official representative) before the study.

The children were split into two groups depending on which technology was used to treat the congenital spinal column curvature.

Group 1, 6 patients: abnormal vertebrae were removed though the anterolateral or posterior approach. The local congenital deformity was treated by placing a dorsal metal structure directly upon the spine while also performing local anterior fusion and posterior spondylodesis at the level of the deformed vertebral-motor segment, see Figure 1.

Group 2, 7 patients. Treatment consisted in placing single costocostal metal structures $(n=4)$ or costovertebral structures $(n=3)$ while also performing osteotomy of fused ribs if necessary. Further treatment consisted in staged surgery for mediated treatment of the congenital spinal deformity to improve torso balance and increase the hemithoracic volume on the concave side of the curvature. Fusion and spondylodesis were not performed in these patients, see Figure 2.

Treated patients were followed up and exposed to checkups and scanning every 6 months in the postoperative period. Xrays were used to estimate the spinal deformity and the position of metal structure supports. Scoliotic and kyphotic deformations were assessed by the Cobb method. CT data were used to verify correct posture of the spinal structure supports, to estimate the hemithorax size, and to evaluate the bone fusion rate in the vertebral motor segments where spinal systems were used. The lung volume was measured by the Philips software; tissue was segmented and cut off in a $3 \mathrm{D}$ reconstruction for further calculation. 


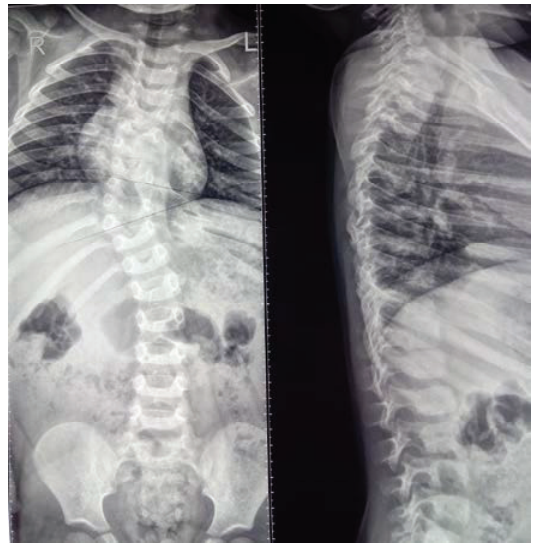

$A$

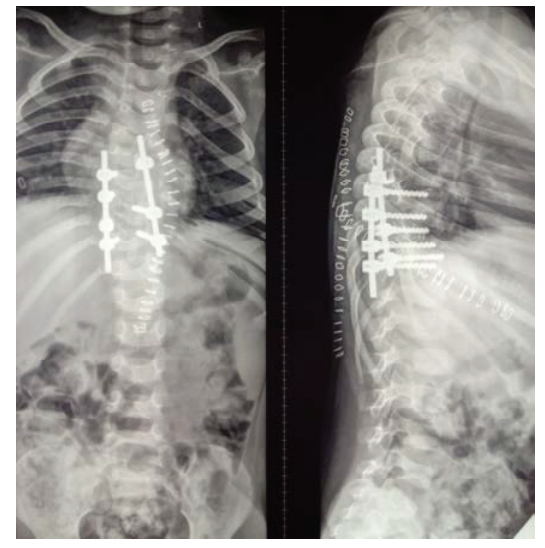

$B$
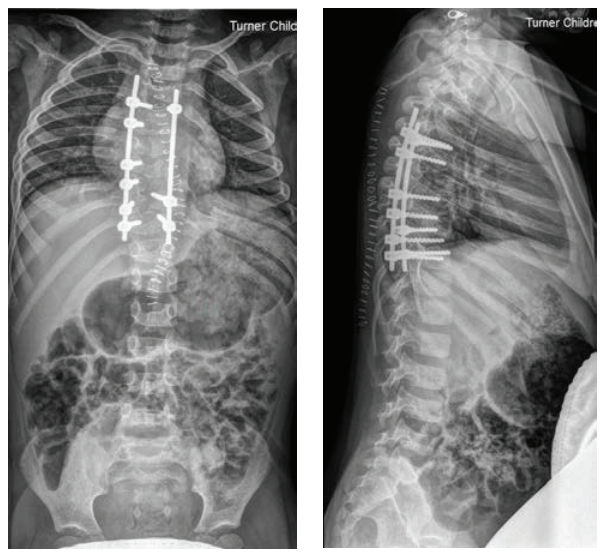

C

Fig. 1. X-ray, Patient Y., aged 2 years 9 months, Group 1. Congenital thoracic scoliosis, posterolateral hemivertebra Th9(D), butterfly vertebra Th5, abnormal segmentation of lateral body surfaces in Th7 to Th10 (S), unilateral costal synostosis (S): A - before surgery; B-after removing the posterolateral hemivertebra Th9(D) and implanting a multi-support metal structure to fix the deformity; $C$-after removing the butterfly vertebra and fixing the deformity with a multi-support transpedicular system

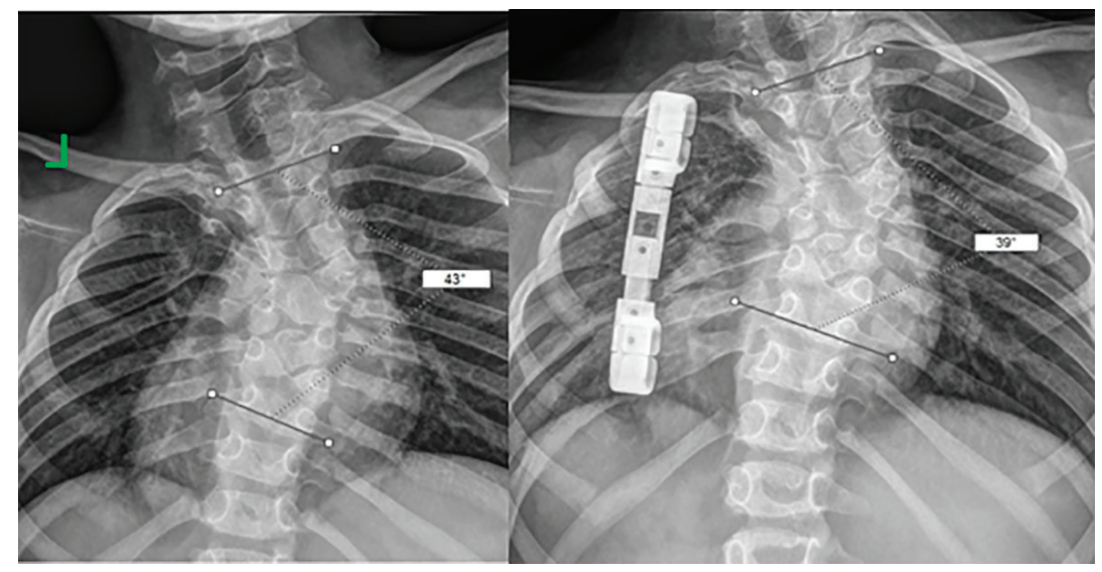

$A$

$B$

Fig. 2. X-ray of a Group 2 patient before surgery (A) and one year after surgery (B) 
Volume was measured for the lung on the concave deformity side; the total lung volume was measured as well. The data thus obtained were processed statistically.

If there were any indications for that, a patient was subject to the next surgical treatment stage according to the method (the removal of the abnormal vertebrae with local deformity treatment; or distraction of the costocostal system) and their specific spinal deformity. Each patient was followed up for 3 years thereafter.

\section{RESULTS}

In all patients, the spinal deformity was due to multiple malformations (butterfly vertebrae, lateral and posterolateral hemivertebra, unsegmented bars, unilateral costal synostosis) in various combinations. Patients were aged $3.6 \pm 0.98$ years in Group $1,5 \pm 1.4$ years in Group 2. Before surgery, scoliotic deformity averaged $39.7^{\circ}$ $\pm 25.1^{\circ}$, kyphosis averaged $26.7^{\circ} \pm 4.6^{\circ}$ in Group $1 ; 69.7^{\circ} \pm 21.7^{\circ}$ and $32.3^{\circ} \pm 13.5^{\circ}$, respectively, in Group 2. The scoliotic deformity was greater in Group 2, as these patients were older, i.e. their deformity had progressed further. Total kyphosis was approximately the same in both groups and within the normal physiological range. The lung volume totaled $972.8 \pm 339.1$ and $990.6 \pm 399 \mathrm{~cm}^{3}$ in Groups 1 and 2, respectively. Before surgery, the lung volume on the concave deformity side was $465.0 \pm$ $144.9 \mathrm{~cm}^{3}(47.8 \%$ of TLV) in Group 1 , $479.7 \pm 203.8 \mathrm{~cm}^{3}(48.4 \%$ of TLV) in Group 2. After staged surgery, scoliotic deformity averaged $14.4^{\circ} \pm 11.9^{\circ}$ (reversed by $63.7 \%$ ), and kyphosis averaged $21.7^{\circ} \pm 3.8^{\circ}$ (reversed by $18.7 \%$ ) in Group $1 ; 63.8^{\circ} \pm 16.3^{\circ}$ $(8.5 \%)$ and $29.8^{\circ} \pm 12.2^{\circ}(7.8 \%)$, respectively, in Group 2. The lung volume totaled $994.8+346.1$ and $1,484.2+407.1 \mathrm{~cm}^{3}$ in Groups 1 and 2, respectively. After surgery, the lung volume on the concave deformity side was $487.9 \pm 126 \mathrm{~cm}^{3}$ (49\% of TLV) in Group 1, 691.5 $187.6 \mathrm{~cm}^{3}(46.6 \%$ of TLV) in Group 2, see Figure 3.

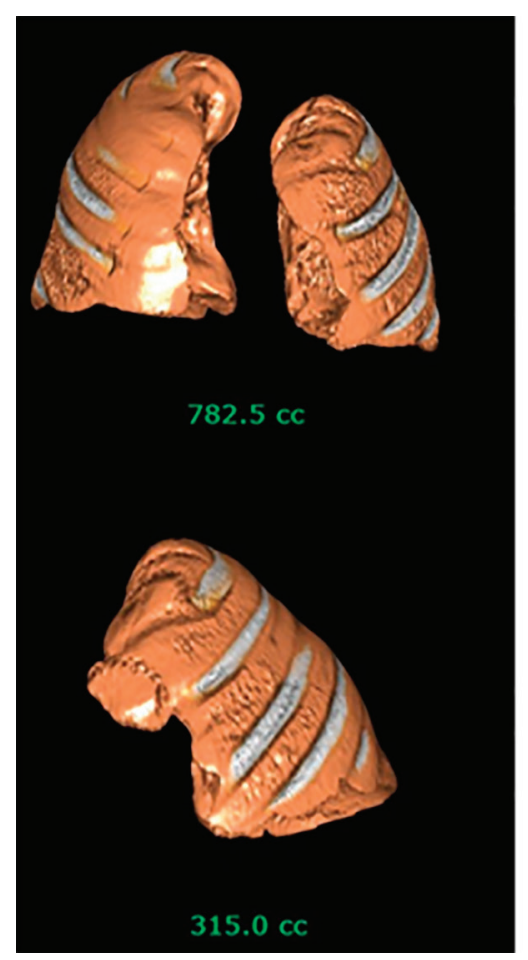

A

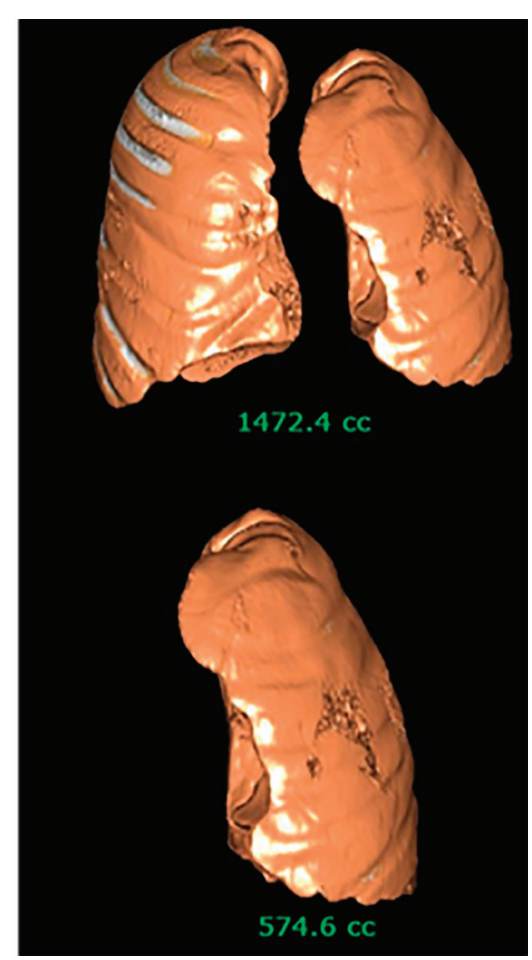

$B$

Fig. 3. 3D reconstruction of a Group 2 patient's lungs, see Figure 2 for X-rays. A: before surgery; $B$ : one year after surgery.

The total lung volume doubled, the implant-side lung volume rose by $45 \%$ 
Group 1 patients had two surgeries on average, while their Group 2 counterparts had three. It should be noted that Group 1 patients had their surgeries upon abnormal vertebrae as the deformity progressed; Group 2 patients had staged repeated surgeries within 6 to 8 months after the first surgery.

Loosening metal structures were detected in 2 patients $(28.6 \%)$ in Group 2, both had a costovertebral system. All the cases required repeated surgery to stabilize the structure.

\section{DISCUSSION}

Choosing the surgical tactic to treat congenital spinal deformities coupled with multiple malformations is a relevant and unresolved issue. The current use of "growing" structures, whether costocostal or costovertebral, is drastically different from the radical local treatment of deformity coupled fixing the vertebral motor segments; the fundamental difference lies in how the two techniques affect the spinal column as well as in their use (or non-use) of arthrodesis. The research data generally shows that prolonged stabilization has an adverse impact on thoracic growth and development; meanwhile, cases of fixing a minimum number of vertebrae are ignored. The use of costocostal or costovertebral corrective implants does not have a universally proven positive effect on external respiration; besides, it is associated with spontaneous bone fusion, negative effects on the frontal and sagittal spine profiles, while resulting in no significant deformity reversal.

This study has shown that in Group 1, where the intervention included removing abnormal vertebrae and fixing as few as possible vertebral motor segments, scoliotic deformity reversal $(63.7 \%)$ and kyphosis reversal $(18.7 \%)$ were far greater than in Group 2 (8.5\% and 7.8\%, respectively). On a separate note, radical local treatment of congenital deformity coupled with using spinal systems to stabilize the vertebral motor segments did not have negative effects on the thoracic organ development and hemithorax volume. These patients had a slightly larger lung volume (TLV and on the concave side of the deformity) several years after the staged surgeries were complete, likely due to growth.

The present study indicates a zero statistically significant impact of this intervention on the TLV-to-CSLV (concave-side lung volume) ratio (percentage-wise). Despite a greater absolute TLV in Group 2, no relative increase in CSLV was noted, while the TLV-to-CSLV ratio (percentage-wise) remained at the pre-surgery levels. Group 2 patients were somewhat older, and the observed increase in the TLV was likely due to their intensive growth typical of children aged 5 to 7 .

\section{CONCLUSIONS}

Thus, the outcomes of the surgical treatment of congenital thoracic spine deformity coupled with multiple vertebral malformations and costal synostosis prove preferable staged surgery performed directly on the spine to remove the bodies of malformed vertebrae, coupled with the radical treatment of local curvature, fixing a minimum number of vertebral motor segments, and osteoplasty. Stabilization must be provided by structures with transpedicular supports; such structures must only be placed on the bodies of the vertebrae adjacent to the malformed vertebra. It should also be noted that spinal systems did not have any notable adverse effects on thoracic development and the lung volume. This initial data proves staged surgery to be feasible and recommendable for fixing the local deformity and stabilizing the vertebral motor segments in patients with congenital spinal curvature and multiple vertebral malformations.

\section{FINANCIAL SUPPORT AND SPONSORSHIP \\ Nil. \\ CONFLICTS OF INTEREST \\ The authors declare no conflict of interest}

\section{REFERENCES}

1. Vissarionov S.V. Surgical treatment of the segmental instability of the thoracic and lumbar spine in children: Doctor of medical sciences dissertation abstract [Khirurgicheskoe lechenie segmentarnoi nestabil'nosti grudnogo i poiasnichnogo otdelov pozvonochnika u detei: avtoref. dis. d-ra med. nauk]. Novosibirsk, 2008, 228 p. 
2. Kaspiris A. Surgical and conservative treatment of patients with congenital scoliosis: a search for long-term results. Scoliosis, 2011, vol. 6, no 1, p. 12, doi 10.1186/1748-7161-6-12.

3. Campbell R.M. Jr., Smith Hell-Vocke A.K. Expansion thoracoplasty: the surgical technique of opening-wedge thoracostomy. Surgical technique. The Journal of Bone and Joint Surgery, 2004, vol. 86, pp. 51-64, doi 10.2106/00004623-200400001-00008.

4. Mehta H.P., Snyder B.D., Callender N.N. et al. The reciprocal relationship between thoracic and spinal deformity and its effect on pulmonary function in a rabbit model. A pilot study. Spine. 2006,vol. 31, no. 23, pp. 2654-2664, doi 10.1097/01.brs.0000244613.66055.b6.

5. Emans J.B., Caubet J.F., Ordonez C.L. et al. The treatment and chest wall deformities with fused ribs by expansion thoracostomy and insertion of vertical expandable prothetic titanium rib: growth of thoracic spine and improvement of long volumes. Spine, 2005, vol. 30, p. 558, doi 10.1097/01.brs.0000175194.31986.2f.

6. Karol L.A., Johnston C.E., Mladenov K. et al. Pulmonary function following early thoracic fusion in non-neuromuscular scoliosis. The Journal of Bone and Joint Surgery, 2008, vol. 90, no 6, pp. 1272-1281, new 10.2106/jbjs.g.00184.

7. Khusainov N.O., Vissarionov S.V., Kokushin D.N. Surgical Treatment Of Children With Congenital Spinal Deformities Associated With Multiple Malformations: A Literature Review. Spine Surgery - Hirurgia pozvonochnika, 2017, vol. 14, no 2, pp. 14-20, doi 10.14531/ss2017.2.14-20.

8. Vissarionov S.V., Husainov N.O., Kokushin D.N. Analysis of results of treatment without-of-spinebased implants in patients with multiple congenital anomalies of the spine and thorax. Pediatric Traumatology, Orthopaedics and Reconstructive Surgery, 2017, vol. 5, no 2, pp. 5-12, doi: 10.17816/PTORS525-12

9. Gadepalli S.K., Hirschl R.B., Tsai W.C. et al. Vertical expandable prosthetic titanium rib device insertion: Does it improve lung function? Journal of Pediatric Surgery, 2011, vol. 46, no 1, pp. 77-80, doi 10.1016/j.jpedsurg.2010.09.070.

10. Vitale M.G., Matsumoto H., Bye M.R. et al. A retrospective cohort study of pulmonary function, radiographic measures, and quality of life in children with congenital scoliosis: an evaluation of patient outcomes after early spinal fusion. Spine, 2008, vol. 33, no. 11, pp. 1242-1249, doi 10.1097/brs.0b013e3181714536.

11. Redding G., Song K., Inscore S. et al. Lung function asymmetry in children with congenital and infantile scoliosis. Spine, 2008, vol. 8, no. 4, pp. 639-644, doi 10.1016/j.spinee.2007.04.020.

12. Lattig F., Taurman R., Hell A.K. Treatment of early-onset spinal deformity (EOSD) with VEPTR: a challenge for the final correction spondylodesis-a case series. Clinical Spine Surgery, 2016, vol. 29, no 5, pp. 246-251, doi 10.1097/bsd.0b013e31826eaf27.

13. Sankar W.N., Acevedo D.C., Skaggs D.L. Comparison of complications among growing spinal implants. Spine, 2010, vol. 35, no 23, pp. 2091-2096, doi 10.1097/brs.0b013e3181c6edd7. 